\title{
Stimulus Propagation and Left Ventricular Torsion
}

\section{Trainini Jorge ${ }^{1 *}$, Elencwajg Benjamín ${ }^{2}$, López Cabanillas Néstor ${ }^{2}$, Herreros Jesús ${ }^{3}$, Lago Noemí ${ }^{4}$, Lowenstein Jorge ${ }^{5}$, Bustamante Munguira Juan ${ }^{6}$ and Trainini Alejandro ${ }^{1}$}

${ }^{1}$ Department of Cardiac Surgery, Hospital Presidente Perón, Argentina

${ }^{2}$ Department of Electrophysiology, Hospital Presidente Perón, Argentina

${ }^{3}$ Department of Cardiovascular and Thoracic Surgery, Universidad Católica San Antonio (UCAM), Spain

${ }^{4}$ Department of Cardiology, Hospital Presidente Perón, Argentina

${ }^{5}$ Department of Echo cardiology, Medical Research, Argentina

${ }^{6}$ Department of Cardiovascular Surgery, University Hospital La Princesa, Spain

Received: 眥 May 28, 2018; Published: 眥 June 11, 2018

*Corresponding author: Jorge Carlos Trainini, Department of Cardiac Surgery, Hospital Presidente Perón, Buenos Aires, Argentina

\begin{abstract}
Introduction and objectives: The ventricular myocardium consists of a continuous muscular band. This anatomy would provide the interpretation for two fundamental aspects of left ventricular dynamics: the mechanism of left ventricular twist and rapid diastolic filling due to the suction effect. The aim of this study was to investigate the electrical activation of the endocardial and epicardial bands to understand ventricular twist and the mechanism of active suction during the diastolic isovolumic phase.

Methods: Five patients underwent three-dimensional endoepicardial electro anatomic mapping during ablation of lone atrial fibrilation or concealed epicardial accessory pathways.

Results: Three-dimensional endo-epicardial mapping demonstrates an electrical activation sequence in the area of the apex loop in agreement with the synchronic contraction of the descending and ascending band segments. The simultaneous and opposing radial activation of the ascending band segment, starting in the descending band segment, in the area in which both band segments intertwine, is consistent with the mechanism of ventricular twist. The late activation of the ascending band segment is consistent with its persistent contraction during the initial period of the isovolumic diastolic phase (the basis of the suction mechanism).
\end{abstract}

Conclusion: This study could explain the process of ventricular twist during systole and the active diastolic suction.

Keywords: Heart; Physiology; Cardiac Electrophysiology; Diastole; Isovolumic diastolic phase

\section{Introduction}

The Torrent Guasp's continuous ventricular myocardial band hypothesis in cardiac mechanics implies a series of associated muscular movements [1]. For this author the ventricular myocardium consists of a continuous muscular band originating in the pulmonary valve and extending to the aortic root, thus limiting the two ventricular chambers (Figure 1). In its spatial trajectory two muscle bands can be identified: the descending endocardial band and the ascending epicardial band, twisted in a double helical coil forming a basal loop (left and right basal segments) and an apical loop (descending and ascending apical segments). In this spatial arrangement, the descending and ascending bands cross each other at a point we will call "segment intersection" (Figure 2). According to this author the movements of left ventricular narrowing, shortening, twisting, lengthening, untwisting and expansion phases during the cardiac cycle would occur by longitudinal diffusion of stimuli along the ventricular myocardial band.

However, this sequential "peristaltic" activation did not correlate with some currently well-known fundamental phenomena, as 
clockwise and counter-clockwise twisting at the left ventricular apex and base, which are mainly responsible for its mechanical efficiency $[2,3]$. In an attempt to explain the mechanism of muscle movements, we investigate the sequence of ventricular electrical activation by means of simultaneous three-dimensional endo-epicardial segment mapping (EAM). Electrical activation is the consequence of the propagation of stimuli through the muscular structure of the heart. The cardiac mechanism of ejection and suction requires structural-functional integration capable of unraveling the different dynamic aspects arising from the propagation of excitation. Many aspects of this theory have been argued, mainly due to the lack of an electrophysiological basis evaluating cardiac activation. The aim of this study was to investigate simultaneously the sequence of electrical activation of the endocardial and epicardial bands of the heart by EAM, investigation has not been evaluated in humans; allowed understand ventricular torsion and the mechanism of active suction during the isovolumic diastolic phase.

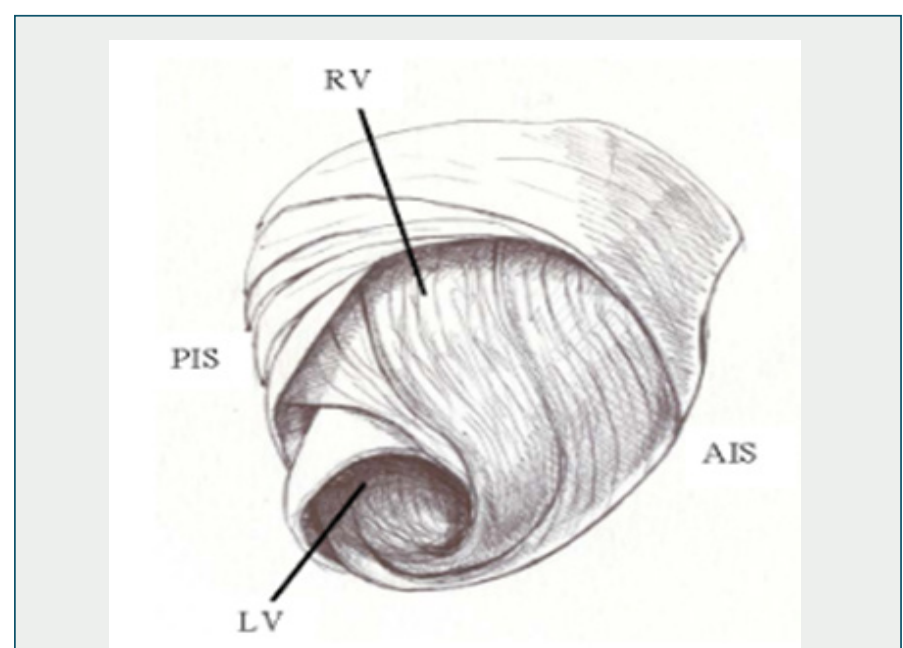

Figure 1: Myocardial muscle band (bovine heart).

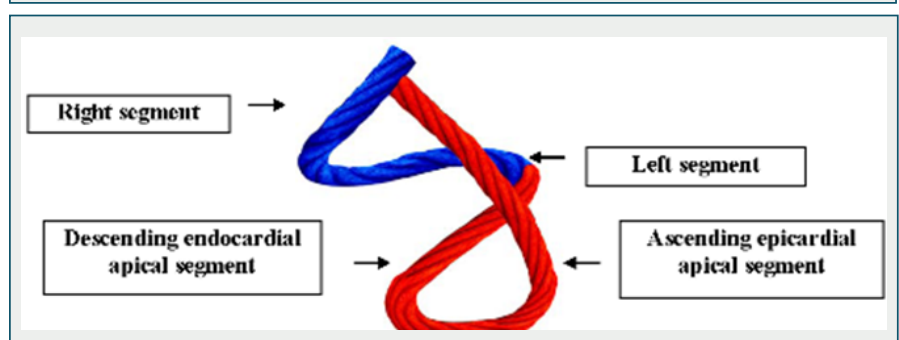

Figure 2: Torrent Guasp's ventricular myocardial band showing its different constitutive segments. Basal loop (blue). Apical loop (red).

\section{Methods}

The study was performed at Hospital Presidente Perón (Buenos Aires, Argentina) and included five patients who had signed an informed consent previously approved by the Institutional Ethics Committee. The study was performed conform the declaration of Helsinki. The left ventricular endo and epicardial electrical activation sequence has been studied using EAM with a navigation system and Carto (Biosense Webster, California, USA) mapping, enabling threedimensional anatomical representation with activation maps and electrical propagation. Isochronic and activation sequence maps were performed, correlating them with surface electrocardiogram. An average of $50 \pm 8$ endocardial and epicardial points were acquired for ventricular activation maps. Apical, lateral and basal views were analyzed. All patients had sinus rhythm. The EAM was performed during the course of radiofrequency ablation for arrhythmias owing to probable abnormal concealed epicardial pathways or lone atrial fibrillation. Mapping was performed at the beginning of the studies, followed by the ablation procedures.

The presence of abnormal pathways did not interfere with mapping, as during the whole procedure baseline sinus rhythm was preserved. As the descending band is endocardial and the ascending band epicardial, two approaches were used to perform mapping. The endocardial access was achieved by conventional atrial transeptal puncture and the epicardial access by a percutaneous approach in the pericardial cavity [4] with an ablation catheter (Navistar TM curve F, Biosense Webster). Endocardial and epicardial mapping was immediately and consecutively performed. They were then superimposed, synchronizing them with electrocardiographic timing. Thus, simultaneous mapping of both ventricles was obtained. Furthemore the propagation times of the electrical activation by the muscular band was measured in milliseconds (ms).

\section{Results}

The EAM took an average of 20 minutes per patient. There were no complications Figures 3-5 illustrate endo and epicardial propagation of electrical activation. In all figures, the left panel shows the right lateral projection and the right panel the simultaneous left anterior oblique projection. The activated areas at each moment are seen in red. The lateral part represents the activation of the bands in Torrent Guasp's rope model [1], where the depolarized zones are shown in red and previously activated zones in refractory period in blue. Below the rope can be seen the average propagation time of electrical muscle band measured in ms on the site analyzed (Table 1). This analysis was performed correlating the path of the stimulus with Torrent Guasp's rope model of the cardiac band.

Left ventricular activation happens in the inter ventricular septum at $12.4 \mathrm{~ms} \pm 1.816 \mathrm{~ms}$ after the start (Figure 3A). Next, following the anatomical arrangement of the descending band it spreads axially towards the ventricular apex. At that moment, the epicardial zone is also activated -the ascending band segmentevidencing radial activation in a region that can be termed as "segment intersection" (Figure 3B). It occurs on average after $25.8 \mathrm{~ms} \pm 1.483 \mathrm{~ms}$ septal stimulation and $38.2 \mathrm{~ms} \pm 2.135$ the onset of cardiac activation. This finding, as later discussed, modifies Torrent Guasp's model and constitutes the electrical foundation of the mechanical phenomenon of ventricular twisting. Synchronously following the anatomical arrangement of the descendent band, activation moves axially towards the ventricular apex reaching the same on average of $58 \mathrm{~ms} \pm 2.0 \mathrm{~ms}$ (Figures $4 \mathrm{~A} \& 4 \mathrm{~B}$ ) and (Table 1). From "segment intersection" the activation loses its unidirectional character and becomes more complex. Figure $4 \mathrm{~A}$ 
shows 3 simultaneous wave fronts: 1) The distal activation of the descending band towards the apical loop; 2) the depolarization of the ascending band from the intersection towards the apex and 3) the activation of the ascending band from the intersection towards the end of the muscle band in the aorta.

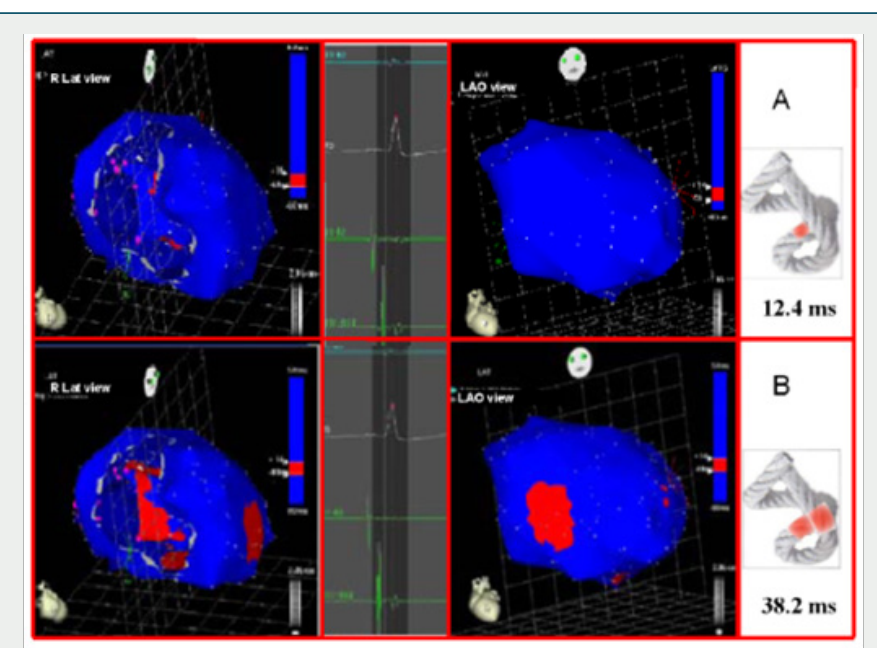

Figure 3: A: Onset of left ventricular activation. The left panel illustrates the depolarization of the ventricular septum, corresponding to the descending band. In the right panel, the ventricular epicardium (ascending band) has not been activated yet. B. Simultaneous band activation. The activation progresses in the left ventricular septum along the descending band (axial activation) and at the same time propagates to the epicardium (radial activation), activating the ascending band.

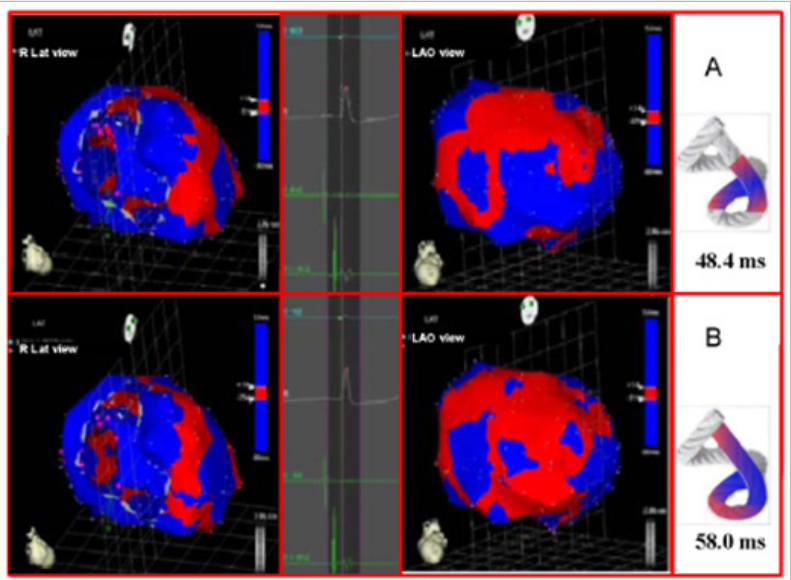

Figure 4: A: Bidirectional activation of the apex and the ascending band. The final septal activation is seen propagating towards the apex, synchronously with the epicardial activation in the same direction. At the same time, the epicardial activation propagates towards the base of the left ventricle. B: Propagation progress. The activation progresses in the directions of the previous figure.

The Figures 4B, 5A \& 5B show the progress and end of this process. In Figure $5 \mathrm{~A}$ it can be seen that endocardial activation finishes much earlier than the end of the QRS period; the rest of the
QRS period corresponds to the late activation of the distal portion of the ascending band, explaining its persistent contraction during isovolumic diastole, which is the basis of the ventricular suction mechanism (Figure 5B). Figure 6 (upper panel) summarizes the stimulation of this rope model found in this investigation.

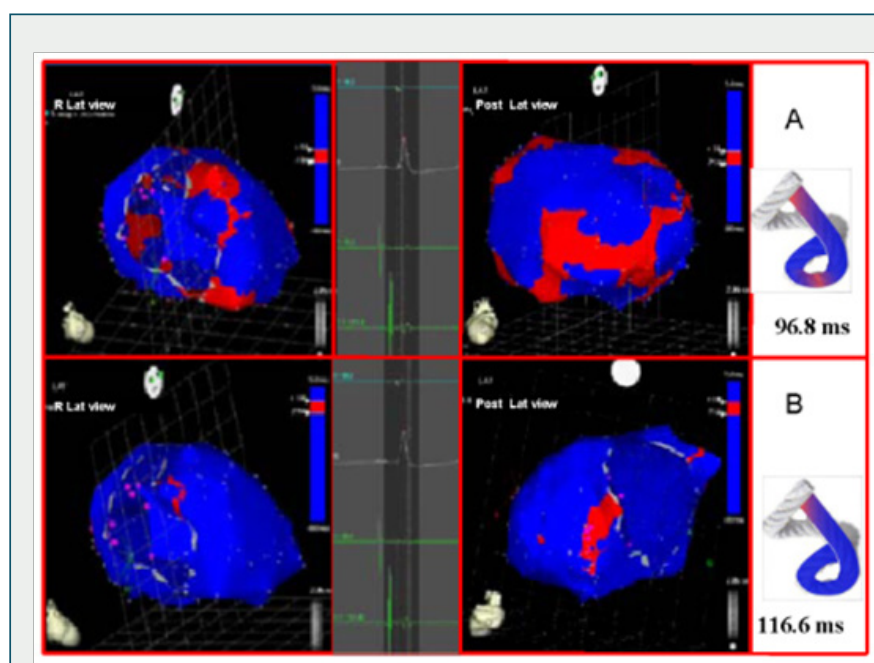

Figure 5: A: Late activation of the ascending band. At this moment, corresponding to approximately $60 \%$ of QRS duration, endocardial activation (descending band) has already been completed. The distal portion of the ascending band (epicardial) depolarizes lately. This phenomenon correlates with its persistent contraction at the initial phase of diastole. B: Final activation. In the right panel, the projection was modified from left anterior oblique to left postero-lateral, showing the very late activation of the distal portion of the ascending band.

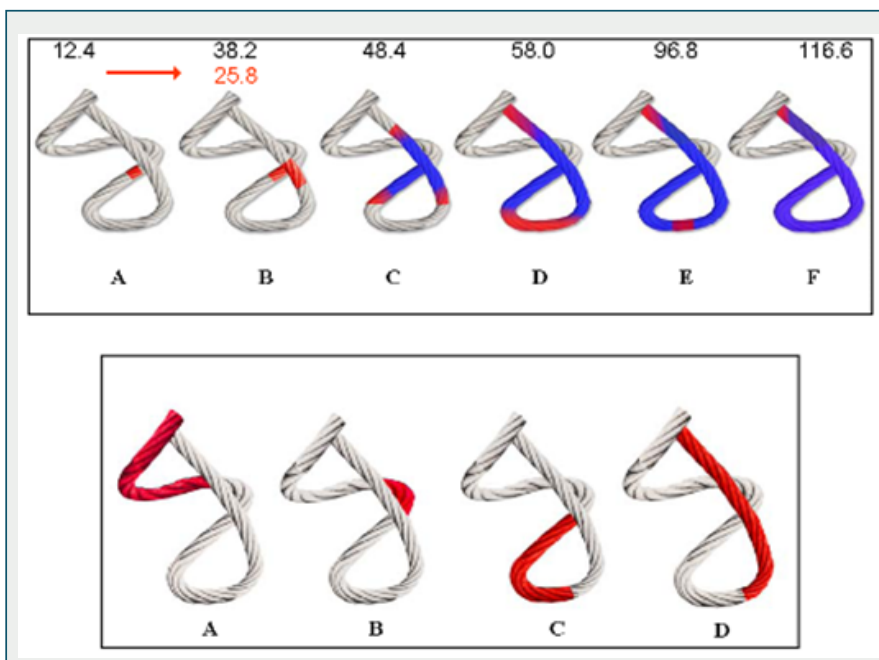

Figure 6: Rope model. Upper Panel: Activation sequence of ventricular myocardial band (A-F) according to our findings. The propagation times are seen in the band and radial delay between the descendent and ascendent segments in milliseconds. Depolarization (red); repolarization (blue). Lower Panel: Unidirectional propagation of excitation wavefront (red) in the ventricular myocardial band according to Torrent Guasp (A-D). 
Table 1: Activation times (ms).

\begin{tabular}{|c|c|c|c|c|c|c|c|}
\hline Localization & Patient & Patient & Patient & Patient & Patient & $\mathbf{X}$ & ds \\
\hline Figure 3A & 10 & 12 & 13 & 15 & 12 & 12.4 & 1.816 \\
\hline Figure 3B & 35 & 38 & 37 & 41 & 40 & 38.2 & 2.135 \\
\hline Figure 4A & 45 & 47 & 49 & 52 & 49 & 48.4 & 2.332 \\
\hline Figure 4B & 55 & 59 & 57 & 61 & 58 & 58 & 2 \\
\hline Figure 5A & 94 & 98 & 98 & 99 & 95 & 96.8 & 1.939 \\
\hline Figure 5B & 115 & 118 & 114 & 120 & 116 & 116.6 & 2.154 \\
\hline
\end{tabular}

References : ms: milliseconds; x: average; ds: standard deviation

\section{Discussion}

This initial study could explain fundamental aspects of left ventricular dynamics:

a) The mechanism of ventricular torsion;

b) The physiology of rapid diastolic filling due to the suction effect.

\section{Stimulus propagation and left ventricular torsion}

In 1915, Thomas Lewis [5] had established that the stimuli arriving along the bundle of His are transmitted through the ventricular walls in an endocardial-epicardial direction, making the papillary muscles the first electrically activated structures. However, J. Robb and R. Robb posed in 1942 a fundamental question: "How is it possible that the transmission of electrical impulses occurs, as all electrical data indicate, from the endocardial to the epicardial surface, given that the ventricular wall is composed of well differentiated bundles separated by sheaths of collagen tissue? " These same authors in dissection studies performed in $1936 \mathrm{had}$ already supported that propagation runs longitudinally (axially) and not transversally, as defined by the classical view of Lewis with these statement: " These data indicate that the excitatory process is conducted axially in the muscles studied along a pathway parallel to fiber direction " [6] Towards 2001, Buckberg and Torrent Guasp ratified the hypothesis that excitation spreads unidirectionally along the ventricular myocardial band Figure 6, (lower panel) [7].

In this experience we found a stimulus trajectory different from that described by Buckberg and Torrent Guasp [7], but which explains the twisting phase of the heart, defined as opposing rotational movement of the base and apex. At the point of the bands intersection the activation propagates from the descending to the ascending band (radial propagation) [3,8]. From this point onwards, the ascending band depolarizes in two senses: towards the apex and towards the base, at the same time that the descending band completes its activation towards the apex (Figure 5). Thus, two essential phenomena occur:

a) As the apical loop is activated from the band intersection in two simultaneous wave fronts (from the descending and from the ascending bands) it generates their synchronized contraction. b) The activation of the ascending band propagates from band intersection in two opposite directions: towards the apex and towards the base (Figure 5). The resulting mechanical contraction will also have a divergent direction, giving origin to the apical and basal clockwise and counter-clockwise rotations, respectively.

It is therefore necessary to find a relationship between activation and the mechanical outcome $[9,10]$. The explanation is provided by the simultaneous axial and radial electrical conduction when it reaches the band intersection, also confirmed by the spatial arrangement of fibers, with subendocardial fibers on the right side and sub epicardial fibers on the left [11]. The isovolumetric systolic phase is produced by the contraction of the basal right and left loops segments. The overlapping shortening phase is due at descend of the base, at the same time as twisting occurs, which is produced longitudinally, as the base contracts before the apex. The fact that the apex remains fixed, is due to the movement of the base, descending in systole and ascending in diastole [12]. This is explained better because the ascending band, rigid in systole and at the beginning of diastole, acts as a tight tutor keeping the apex immobile. The pressure generated to eject the highest amount of blood at the onset of ejection during an interval lasting $20 \%$ of the systolic phase is feasible due to the twisting movement. This action is achieved because the electrical stimulation propagates towards the descending band (axial propagation) and simultaneously to the ascending band (radial propagation). Although the electrical conduction progresses along the ventricular myocardial band, radial propagation towards the ascending band plays an essential role in ventricular twisting by allowing opposing forces on its longitudinal axis, generating the necessary intra ventricular pressure to achieve an abrupt blood ejection. The inter band fibers that cross from the descending band to the ascending band would be responsible of impulse transmission between the bands. Thus, a twisting mechanism similar to "wringing a towel" would be produced $[12,13]$.

Buckberg declared that "the sequence of ventricular muscle entry into activity in the different ventricular regions takes place along the band" [7] similarly to a peristaltic movement Figure 6, (lower panel). This concept remained unchanged until the studies performed in the present investigation, based on the hypothesis that the axial transmission of the stimulus along the ventricular myocardial band supported by Torrent Guasp and Buckberg did not explain satisfactorily the twisting and untwisting movement indispensable to understand cardiac mechanics. Then, how could the ventricle achieve its twisting movement, since this action requires two opposing forces at the same time? Unidirectional activation does not explain twisting or the evolutionary-structural development designed to apply a force capable of ejecting the ventricular content at a speed of $300 \mathrm{~cm} / \mathrm{s}$ at low energetic cost. This is understood by the simultaneous axial and radial activation we have found Figure 6, (upper panel). 


\section{Active suction in the isovolumic diastolic phase}

We have found that the endocardium depolarizes completely during the first part of the QRS (Figure 5A). During the initial part of the isovolumetric diastolic phase, the ascending band remains contracted as a consequence of the depolarization that took place during the last part of the QRS. The explanation of this late contraction does not require depolarization's after the QRS. In our investigation, the final part of the QRS corresponds to the activation of the ascending band (Figure 5B), resulting in the contraction needed to generate suction "plunger effect" during the isovolumic diastolic phase. With the beginning of ventricular untwisting during the iso volumetric diastolic phase, progressive lengthening of the ascending band segment generates a negative intra ventricular pressure while the ascending band segment is still contracted (active process), a residual energy of the torsion process $[14,15]$.

During cardiac resynchronization therapy, we measured intra ventricular pressure to achieve evidence of improved suction mechanism in the isovolumic diastolic phase. Figure 7A shows the left ventricular pressure curve in a patient with left bundle branch block before implanting a cardiac resynchronization therapy device. The hypothesis is that left bundle branch block modifies left ventricular activation sequence and, in consequence, mechanical sequence is also impaired. Thus, the sequential activation of the band segments is also impaired and the suction mechanism is lost. Consequently, diastolic pressure increases. Stimulation of the left ventricular endocardium (the area corresponding to crossing of band segments) in cardiac resynchronization therapy would reestablish the normal electrical activation and mechanical sequence of both band segments. The suction mechanism would also be restored and left ventricular diastolic pressure would decrease (Figure 7B), improving heart failure symptoms.

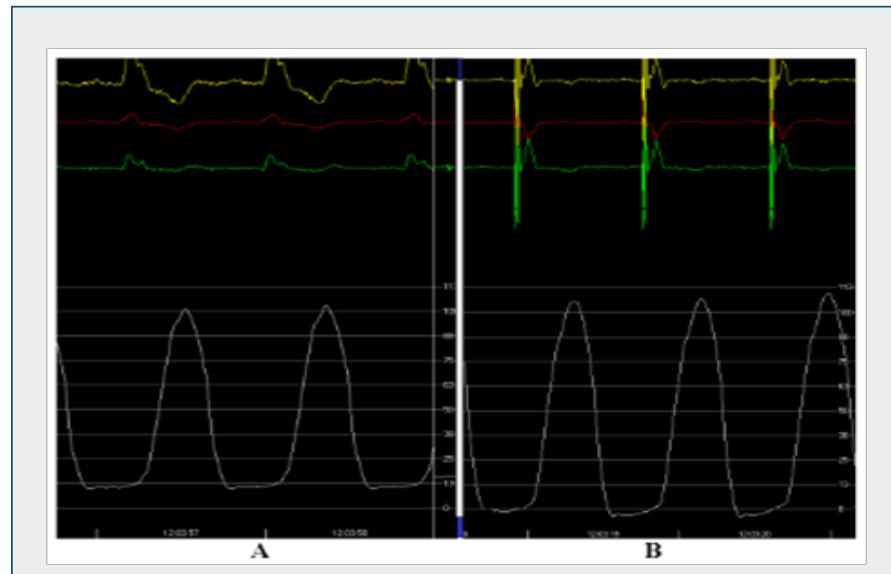

Figure 7: A: Intracardiac pressures. A: Left ventricular presure before resynchronization. B: In the same patient, a decrease in diastolic pressure is seen while the ventricles are resynchronized.

\section{Study limitations}

Torrent Guasp's unique continuous muscular band model is not universally accepted by the scientific community. It should be noted that finding a sequence of ventricular activation is not equivalent to demonstrating the existence of a single myocardial band. The anatomical conformation of the physiological conduction pathways of the electrical stimulus could be responsible for these activation sequences, without the need for a myocardial band with a specific disposition. The results of the present study are clear but the low number of patients is a limitation. Yet, the physiological process do not need a high number of experiences as in clinical studies. In addition, these results could be reinterpreted with studies with a larger number of cases or with experimental studies in animals with better control of variables. Despite this may be a limitation, the fact that this study was performed in humans and not in animals makes it more reliable.

Another aspect to consider is that the discussion could be considered speculative, as anatomic or hemodynamic interpretations were based on an electrophysiology study without performing morphological dissections or measuring pressures, volumes or dimensions. In fact, it should be mentioned that Torrent Guasp conducted the first phase of this experience with anatomical data and that Buckberg's interpretations were based on pressure measurements. However, it was necessary to prove the electrophysiological aspect to determine a new cardiac physiology, as the direction of electrical conduction determines the real function. All these considerations were cleared up in the present study and should be continued in future investigations.

\section{Conclusion}

The data obtained are especially important, as they were recorded in humans, in physiological conditions. According to these findings, we may conclude:

a) Three-dimensional endo-epicardial mapping demonstrates an electrical sequence of activation in the area of the apical loop in agreement with the synchronic contraction of the descending and ascending band segments.

b) The simultaneous and opposing activation of the ascending band segment from the starting point of its radial activation from the descending band segment is consistent with the simultaneous reverse rotation of the apical and basal areas (mechanism of ventricular torsion).

c) The late activation of the ascending band segment is consistent with its persistent contraction during the initial period of isovolumic diastole (untwististing and suction, and takes place without need of postulating further electrical activations after the QRS complex).

\section{References}

1. Torrent Guasp F (1998) Structure and function of the heart. Rev Esp Cardiol 51(2): 91-102.

2. Brecher GA (1956) Experimental evidence of ventricular diastolic suction. Circ Res 4: 513-518.

3. Trainini JC, Herreros J, Cabo J, Otero Coto E, Cosín Aguilar J (2011) The cardiac pump suction. Application of the myocardial band of Torrent 
Guasp to the surgical treatment of heart failure. Cir Cardiovasc 18: 103112.

4. Sosa E, Scanavacca M, d'Avila A, Pilleggi F (1996) A new technique to perform epicardial mapping in the electrophysiology laboratory. J Cardiovasc Electrophysiol 7(6): 531-536.

5. Lewis T, Rothschild MA (1915) The excitatory process in dog's heart. IIThe ventricles. Philos R Trans Soc 206: 325-334.

6. Robb JS, Robb RC (1942) The normal heart: Anatomy and physiology of the structural units. Am Heart J 23(4): 455-467.

7. Buckberg GD, Coghlan HC, Torrent Guasp F (2001) The structure and function of the helical heart and its buttress wrapping. V. Anatomic and physiologic considerations in the healthy and failing heart. Semin Thorac Cardiovasc Surg 13(4): 358-385.

8. Torrent Guasp F, Buckberg G, Carmine C, Cox J, Coghlan H, et al. (2001) The structure and function of the helical heart and its buttress wrapping. I. The normal macroscopic structure of the heart. Semin Thorac Cardiovasc Surg 13(4): 301-319.

9. Poveda F, Gil D, Martí E, Andaluz A, Ballester M, et al. (2013) Tractographic study of the helical anatomy of the ventricular myocardium by means of magnetic resonance by diffusion tensor. Rev Esp Cardiol 66: 782-790.
10. Cosín Aguilar J, Hernándiz Martínez A (2013) The disposition of myocardial fibers in a band conditions the morphology and function of the heart. Rev Esp Cardiol 66: 768-770.

11. Sallin EA (1969) Fiber orientation and ejection fraction in the human left ventricle. Biophys J 9(7): 954-964.

12.Zarco $P$ (2001) The ventricular rapid filling phase: a muscle relaxation or contraction process? Rev Esp Cardiol 54(9): 1031-1032.

13. Ashikaga H, Coppola BA, Hopenfeld B, Leifer ES, Mc Veight ER, et al. (2007) Transmural dispersion of myofiber mechanics: Implications for Electrical Heterogeneity In Vivo. J Am Coll Cardiol 49: 909-916.

14. Sonnenblick EH (1980) The structural basis and importance of restoring forces and elastic recoil for the filling of the heart. Eur Heart J 1(1): $107-$ 110.

15. Herreros J, Trainini JC, Bernal JM, Gutiérrez F, Cabo J, et al. (2011) Treatment of heart failure: new therapeutic strategies. Circ Cardiov 18: 113-120.

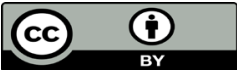

This work is licensed under Creative Commons Attribution 4.0 License

To Submit Your Article Click Here:

Submit Article

DOI: 10.32474/ACR.2018.01.000109

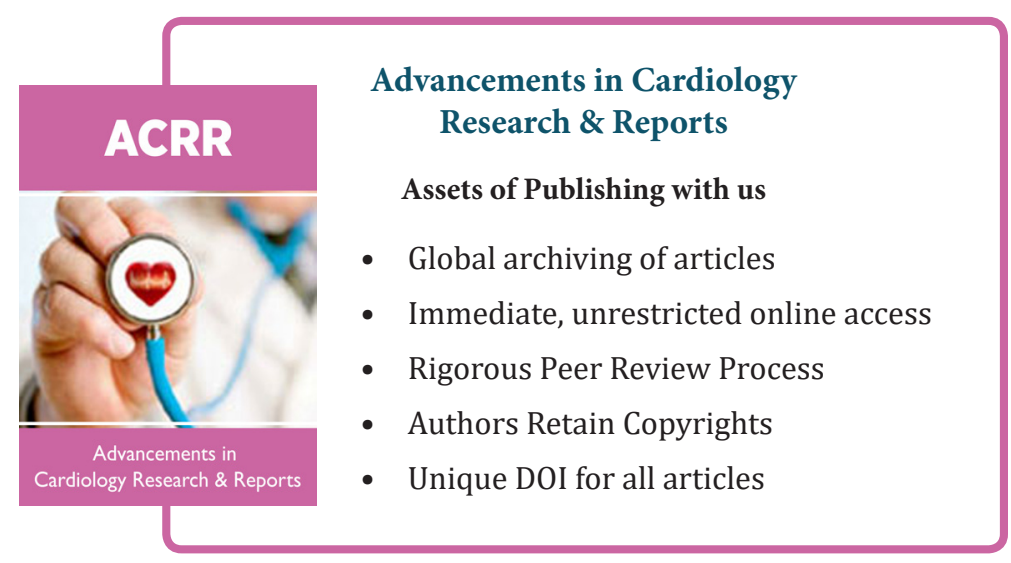

